\title{
Non-standard AGE4 epitopes that predict polyneuropathy independently of obesity can be detected by slot dot-blot immunoassay
}

\author{
Agnieszka Bronowicka-Szydełko, ${ }^{1, A, D}$, Małgorzata Krzystek-Korpacka ${ }^{1, C, D}$, Aleksandra Kuzan ${ }^{1, C}$, Kinga Gostomska-Pampuch ${ }^{1,2, C}$, \\ Małgorzata Gacka ${ }^{3, B}$, Urszula Jakobsche-Policht ${ }^{3, B}$, Rajmund Adamiec ${ }^{3, D, E}$, Andrzej Gamian ${ }^{1,2, D, F}$ \\ ${ }^{1}$ Department of Medical Biochemistry, Wroclaw Medical University, Poland \\ ${ }^{2}$ Laboratory of Medical Microbiology, Hirszfeld Institute of Immunology and Experimental Therapy, Polish Academy of Sciences, Wrocław, Poland \\ ${ }^{3}$ Department of Angiology, Diabetes and Hypertension, Wroclaw Medical University, Poland \\ A - research concept and design; B - collection and/or assembly of data; C - data analysis and interpretation; \\ $D$ - writing the article; $E$ - critical revision of the article; $F$ - final approval of the article
}

Address for correspondence

Agnieszka Bronowicka-Szydełko

E-mail:agnieszka.bronowicka-szydelko@umed.wroc.pl

Funding sources

The study was supported by National Science Centre with a grant No. 2012/05/N/NZ5/00836.

Conflict of interest

None declared

Received on November 8, 2018

Reviewed on December 3,2018

Accepted on September 25, 2019

Published online on February 4, 2020

Cite as

Bronowicka-Szydełko A, Krzystek-Korpacka M, Kuzan A, et al. Non-standard AGE4 epitopes that predict polyneuropathy independently of obesity can be detected by slot dot-blot immunoassay. Adv Clin Exp Med. 2020;29(1):91-100. doi:10.17219/acem/112612

DOI

10.17219/acem/112612

Copyright

Copyright by Author(s)

This is an article distributed under the terms of the

Creative Commons Attribution 3.0 Unported (CC BY 3.0)

(https://creativecommons.org/licenses/by/3.0/)

\begin{abstract}
Background. Advanced glycation end-products (AGEs) are formed during cascade reactions between reducing sugars or reactive aldehydes and proteins, lipids or DNA molecules. They constitute a group of various stable compounds. Advanced glycation end-products are considered potential biomarkers of metabolic disorders. However, so far only a few methods to determine the level of individual AGEs have been developed.

Objectives. The aim of the study was to compare the efficiency of the slot-dot blot method and direct enzyme-linked immunosorbent assay (ELISA) in detecting non-standard epitopes of methylglyoxal (MGO)modified proteins (AGE4) found in diabetes serum in trace amounts, and to assess AGE4 in diabetes and associated metabolic abnormalities.
\end{abstract}

Material and methods. The presence of AGE4 was detected using 2 methods: direct ELISA and the slotdot blot method - a newly developed immunoassay based on monoclonal, commercially available antibody detection of non-standard AGE epitopes. AGE4 quantification, expressed as median AGE4 in arbitrary units (AU) and AGE4 positivity (the percent of samples with detectable AGE4) was related to diabetes-associated metabolic abnormalities, complications and treatment.

Results. Slot-dot blot was significantly more efficient than ELISA in detecting non-standard AGE4 epitopes. AGE4 positivity was less frequent in patients with microangiopathy and in those with polyneuropathy. In patients with abnormal glucose metabolism, metformin treatment was associated with higher AGE4. AGE4 positivity was significantly lower in gliptin-treated patients. Multivariate analysis showed that polyneuropathy and obesity were independently associated with AGE4 positivity, with odds ratios (ORs) of 0.21 and 3.02, respectively. Moreover, logistic regression showed that AGE4 positivity and HbA1c are independent predictors of polyneuropathy. Considering both indicators allows correct classification of $70.4 \%$ of cases with a general accuracy of $76 \%$.

Conclusions. The slot dot-blot method detects compounds found in serum in trace amounts. Accumulation of AGE4 was associated with glucose metabolism abnormalities. A tendency toward AGE4 positivity was less frequent in patients with microangiopathy and in non-treated and gliptin-treated diabetes patients.

Key words: ELISA, diabetes, methylglyoxal, advanced glycation end-products (AGEs), slot dot-blot 


\section{Introduction}

Advanced glycation end-products (AGEs) play a pivotal role in the development of complications in individuals with diabetes. ${ }^{1}$ They have recently gained attention as prospective biomarkers and targets for pharmacotherapy for diabetes, atherosclerosis and related metabolic abnormalities. They are also being investigated as probable contributors to the pathogenesis of neurodegenerative diseases and cancer. ${ }^{2}$ Advanced glycation end-products are formed as a result of non-enzymatic reaction between reducing carbohydrates or oxo-aldehydes and amino groups of proteins, nucleic acids or lipids. Glycation negatively affects the functionality of modified macromolecules. It is a multi-step cascade prompted by hyperglycemia and oxidative stress, yielding compounds of varying structures and stability. ${ }^{3}$ Glycation usually affects proteins with a long half-life, such as extracellular matrix proteins, myelin sheath proteins, crystallins in the eye, or serum albumins. ${ }^{4}$ Proteins with a short half-life also undergo glycation. However, the resulting products are not AGEs, but Amadori compounds, such as glycated hemoglobin $\left(\mathrm{HbA}_{1} \mathrm{c}\right)$, a routinely assessed marker of glycemic control. ${ }^{5}$

Studies on the importance of AGEs in human pathology and potential applications of AGEs in diagnostics and therapy are hindered by their heterogeneity and difficulties in quantification. Only a limited number of AGE structures have been identified, characterized and evaluated in a clinical context. At present, $\mathrm{N}^{\varepsilon}$-carboxymethyl-lysine (CML) is the most extensively studied AGE, followed by $\mathrm{N}^{\varepsilon}$-(1-carboxyethyl)-lysine (CEL), pentosidine, argpyrimidine (AP), methylglyoxal (MGO)-derived hydroimidazolone, glyoxal-derived hydroimidasolone, and MOLD (MGO-lysine dimer). ${ }^{6}$ The relevance of other AGEs remains largely unknown. ${ }^{7}$ The detection and quantification of AGEs in easily available biological material, such as blood, is difficult. They are characterized by low solubility combined with high viscosity, and unlike in tissues, AGEs in blood are present in trace amounts. Therefore, sophisticated instrumental techniques, such as mass spectrometry, are employed to detect specific AGE epitopes. ${ }^{8}$ Moreover, extraction of AGEs from sera using affinity or liquid chromatography is frequently required prior to their identification and quantification. ${ }^{9}$ The necessity of advanced equipment and specialists limits the possibilities of applying these techniques in clinical practice on a large scale. More accessible techniques such as immunoassays are used mostly to quantify so-called "total AGEs", a pool of various AGEs with fluorescent properties. Although useful for getting a general overview of the degree of glycation, "total AGEs" do not encompass all AGEs (non-fluorescent species are not accounted for), nor discern the concentrations of any specific AGE. This makes the assessment of their individual contributions to a pathology impossible. Data on specific AGEs determined using immunoassays is scanty, and limited mostly to CML and pentosidine. ${ }^{10}$
Methylglyoxal is a low-molecular-weight reactive aldehyde formed endogenously as a by-product of glycolysis and lipid peroxidation. ${ }^{11}$ It evokes inflammatory ${ }^{12}$ and immune responses, ${ }^{13}$ and contributes to mitochondrial dysfunction and the formation of both AGEs and advanced lipoxidation end-products (ALEs). Methylglyoxal has been implicated in the development of complications in diabetes patients ${ }^{13}$ as well as those with cancer ${ }^{14}$ and multiple sclerosis. ${ }^{15}$ The presence of MGO-modified proteins is usually detected indirectly, by evaluating the titer of the respective autoantibodies. Hydroimidazolone-1 (MG-H1) is the only known specific epitope formed on MGO-modified proteins. Others, namely CML and CEL, can be formed not only by MGO but also by glucose modification. Hydroimidazolone-1 has recently been proposed as a biomarker of reduced diastolic heart function in type 1 diabetes mellitus (T1DM). ${ }^{16}$

To our knowledge, no immunoassay allowing quantification of MGO-derived epitopes (AGE4) other than CML, CEL or MG-H1 has been published. In this paper, we therefore present a slot dot-blot method for determining MGOderived non-standard epitopes using commercially available monoclonal antibodies. The suitability of the assay for patient evaluation was tested on individuals with diabetes and related metabolic abnormalities and complications.

\section{Material and methods}

\section{Serum samples}

Serum samples were obtained from clotted (15 min, room temperature) and centrifuged ( $15 \mathrm{~min}, 400 \times \mathrm{g}$ ) blood drawn by venipuncture following a 12-hour overnight fast and collected into serum-separator tubes.

For the purpose of validation of the method performance in a clinical setting, serum samples were obtained from 46 individuals, of whom 26 were diagnosed with diabetes (10 men and 16 women), and the remaining 20 were apparently healthy blood donors (10 men and 10 women). Similarly, AGE4 was determined in 131 individuals: 91 with abnormalities in glucose metabolism (85 diagnosed with diabetes) and 30 apparently healthy blood donors.

Patients with deregulated glucose metabolism were recruited from the Department of Angiology, Diabetes and Hypertension of Wroclaw Medical University (Poland). Apparently healthy blood donors, without known glucose metabolism abnormalities, were recruited from the Regional Center of Blood Donation and Therapy (Wrocław, Poland) using the following inclusion criteria: age $>50$ years; no known systemic disease, dementia or depression; no ongoing inflammation; and fasting glucose $<100 \mathrm{mg} / \mathrm{dL}$.

Diabetes was diagnosed using the World Health Organization (WHO) criteria. The disease was considered uncontrolled if $\mathrm{HbA}_{1} \mathrm{c}$ exceeded 6.4\%. ${ }^{17}$ Overweight was defined according to WHO classifications as body mass index (BMI) 
$25-30 \mathrm{~kg} / \mathrm{m}^{2}$, and obesity as BMI $\geq 30 \mathrm{~kg} / \mathrm{m} .{ }^{2,18}$ Metabolic syndrome (MetS) was diagnosed using the International Diabetes Federation (IDF) criteria ${ }^{19}$ as the coexistence of central obesity plus any 2 of the following: hypertriglyceridemia ( $\geq 150 \mathrm{mg} / \mathrm{dL}$ ) or receiving treatment, low highdensity lipoprotein (HDL)-cholesterol ( $<40 \mathrm{mg} / \mathrm{dL}$ in men and $<50 \mathrm{mg} / \mathrm{dL}$ in women), hypertension $(\geq 130 \mathrm{~mm} \mathrm{Hg}$ for systolic or $\geq 85 \mathrm{~mm} \mathrm{Hg}$ for diastolic) or receiving treatment, glucose $\geq 100 \mathrm{mg} / \mathrm{dL}$ or diagnosed with diabetes and receiving treatment. Hyperlipidemia was diagnosed using the guidelines of the European Society of Cardiology (ESC) and the European Atherosclerosis Society (EAS) ${ }^{20}$ as elevated triglycerides ( $\geq 150 \mathrm{mg} / \mathrm{dL})$ and/or low-density lipoprotein (LDL)-cholesterol ( $\geq 115 \mathrm{mg} / \mathrm{dL})$ and/or total cholesterol ( $\geq 190 \mathrm{mg} / \mathrm{dL}$ ), or receiving treatment. Hyperuricemia was diagnosed using the National Health and Nutrition Examination Survey III (NHANES-III) criteria ${ }^{21}$ as uric acid $\geq 7.0 \mathrm{mg} / \mathrm{dL}$ in men and $\geq 5.7 \mathrm{mg} / \mathrm{dL}$ in women.

Macroangiopathy (atherosclerosis of the arteries) was diagnosed if any of the following was present: myocardial infarct, stroke, percutaneous coronary intervention, coronary artery bypass graft, acute coronary syndrome, objective evidence of coronary artery disease (defined as a positive exercise test and angiography with at least 1 stenosis $>50$ ), symptomatic peripheral arterial obstructive disease (confirmed by an ankle/brachial pressure index $<0.90$ or an amputation), stenosis of the carotid artery, or cardiovascular death. Microangiopathy was diagnosed in patients with diabetic nephropathy (albuminuria $>30 \mathrm{mg} / \mathrm{g}$, creatinine and glomerular filtration rate (eGFR) using the Chronic Kidney Disease Epidemiology Collaboration Equation (CKD-EPI)), diabetic retinopathy (microaneurysms, dot-blot hemorrhages, flame-shaped hemorrhages, retinal edema and hard exudates, soft exudates, venous loops and venous beading, intraretinal microvascular abnormalities, hemorrhage into the vitreous, traction retinal detachments, macular edema etc., detected using ophthalmoscopy, fundus fluorescein angiography or optical coherence tomography) or polyneuropathy (diagnosed after excluding other possible causes with the pinprick test to establish absent or reduced pain sensations, electromyography and clinical evaluation using a quantitative diagnostic tool such as the Neuropathy Impairment Score).

The patients' demographic, laboratory and clinical data was collected prospectively and is presented in Table 1. Data on the implemented treatment as well as the co-existence of other abnormalities is given in Table 2 .

\section{Ethical considerations}

The study protocol was approved by the Medical Ethics Committee of Wroclaw Medical University (approvals No. KB-303/2010 and No. KB-384/2012) and was in accordance with the ethical standards formulated in the Helsinki Declaration of 1975. Informed consent was obtained from all participating individuals.
Table 1. Characteristics of patients according to metabolic abnormalities

\begin{tabular}{|l|c|}
\hline \multicolumn{1}{|c|}{ Metabolic abnormalities } & $\mathrm{n}(\%)$ \\
\hline Diabetes & $85(93.4)$ \\
T1DM & $3(3.5)$ \\
T2DM & $76(89.4)$ \\
T3DM & $6(7.1)$ \\
\hline Overweight/obesity & \\
overweight & $28(30.8)$ \\
obesity I/II/II & $37(40.7)$ \\
\hline Macroangiopathy & $69(75.8)$ \\
IHD & $32(35.2)$ \\
ACS & $18(19.8)$ \\
IS & $11(12.1)$ \\
AO & $28(30.8)$ \\
CAP & $62(68.1)$ \\
\hline Microangiopathy & $46(50.5)$ \\
retinopathy & $11(12.1)$ \\
nephropathy & $20(22.0)$ \\
polineuropathy & $35(38.5)$ \\
\hline Hypertension & $83(91.2)$ \\
\hline Hyperlipidemia & $78(85.7)$ \\
\hline Hyperurykemia & $26(33.8)$ \\
\hline FLD & $12(13.2)$ \\
\hline CKD & $10(11.0)$ \\
\hline
\end{tabular}

IHD - ischemic heart disease; ACS - acute coronary syndromes; IS - ischemic stroke; AO - arteriosclerosis obliterans; CAP - carotid artery plaques; FLD - fatty liver disease; CKD - chronic kidney disease.

Table 2. Characteristics of patients according to treatment

\begin{tabular}{|l|c|}
\multicolumn{1}{|c|}{ Treatment } & $\mathrm{n}(\%)$ \\
\hline Antihypertensive drugs & $77(84.6)$ \\
\hline Dyslipidemia medications & $55(60.4)$ \\
\hline Aspirin & $60(65.9)$ \\
\hline Anticoagulants & $13(14.3)$ \\
\hline Clopidogrel & $11(12.1)$ \\
\hline Metformin & $65(71.4)$ \\
\hline Acarbose & $10(11.0)$ \\
\hline Sulphonylureas & $31(34.1)$ \\
\hline Gliptins & $12(13.2)$ \\
\hline Insulin & $38(41.8)$ \\
\hline
\end{tabular}

\section{Antibodies}

Specific anti-AGE4 monoclonal antibodies (clone No. 14B5; Cosmo Bio Co. Ltd., Tokyo, Japan) - i.e., MGOmodified bovine serum albumin (BSA) - were used as the primary antibodies. These antibodies have been shown not to cross-react with glucose-modified BSA (AGE1), glyceraldehyde-modified BSA (AGE-2), glycolaldehydemodified BSA (AGE-3), glyoxal-modified BSA (AGE-5), anti-3-deoxyglucose-modified BSA (AGE-6), CML-modified BSA, CEL-modified BSA, or with native BSA. The method for obtaining and purifying anti-AGE4 monoclonal antibodies was described by Takeuchi et al. ${ }^{22}$ 
Polyclonal goat anti-mouse IgG $(\mathrm{H}+\mathrm{L})$ antibodies conjugated with horseradish peroxidase (HRP) (Jackson ImmunoResearch Europe Ltd., Ely, UK; cat. No. 115-035-166) were used as secondary antibodies at the dilution recommended by the manufacturer $(1: 2,000)$.

\section{Direct ELISA assay}

The wells of flat-bottom Maxisorp microtiter plates (Nunc A/S, Roskilde, Denmark) were coated with $50 \mu \mathrm{L}$ of serum samples diluted in $50 \mathrm{mM}$ carbonate buffer ( $\mathrm{pH}$ 9.6) and incubated for $4 \mathrm{~h}$ at $37^{\circ} \mathrm{C}$, followed by overnight incubation at $4^{\circ} \mathrm{C}$. The following serum dilutions were tested: $\times 200$, $\times 100, \times 50, \times 20, \times 10, \times 5, \times 4, \times 2$, and undiluted. After intensive washing with 3 changes of $300 \mu \mathrm{L}$ of tris buffered saline with tween 20 (TBS-T), pH 7.4, the plates were blocked with $300 \mu \mathrm{L}$ of $5 \%$ skimmed milk for $20 \mathrm{~h}$ at $4{ }^{\circ} \mathrm{C}$. Unbound milk was removed by washing with TBS-T, pH 7.4 $(3 \times 300 \mu \mathrm{L})$, and the plates were incubated for $2 \mathrm{~h}$ at $37^{\circ} \mathrm{C}$ with $50 \mu \mathrm{L}$ of the primary antibodies per well. The following dilutions of the primary antibodies were tested: 1:2,000, 1:1,000, $1: 500$, and 1:250. Subsequently, following a wash step $(3 \times 300 \mu \mathrm{L}$ of TBS-T, $\mathrm{pH} 7.4)$, the plates were treated with the secondary antibodies conjugated with HRP $(50 \mu \mathrm{L}$ per well; $1: 2,000)$ for $1.5 \mathrm{~h}$ at $37^{\circ} \mathrm{C}$. After washing $(3 \times 300 \mu \mathrm{L}$ of TBS-T, pH 7.4), the emptied wells were filled with $50 \mu \mathrm{L}$ of o-phenylenediamine dihydrochloride (OPD) (Thermo Fisher Scientific, Rockford, USA) and incubated for $10 \mathrm{~min}$ at room temperature. The appearance of an orange-brown product, proportional to the AGE4 concentration in serum samples, was measured at $\lambda=450 \mathrm{~nm}$ using an EnSpire ${ }^{\circledR}$ microplate reader (Perkin Elmer Inc., Waltham, USA).

Additionally, the following negative controls were run simultaneously with the samples: antigen control (no antigen), blockage control (without the primary or secondary antibodies), primary antibody control (no secondary antibody), and secondary antibody control (without the primary antibody). All the samples and controls were run in triplicate.

\section{Slot dot-blot assay}

Fifty microliters of twice-diluted sera in phosphatebuffered saline (PBS) pH 7.2 were applied in duplicate to methanol-activated polyvinylidene difluoride membrane (Immobilon ${ }^{\circledR}$-P Transfer Membrane; Bio-Rad Laboratories, Hercules, USA) using slot apparatus (Bio-Rad Laboratories). The prepared membranes were soaked in 5\% skimmed milk in TBS-T for $2 \mathrm{~h}$ at room temperature, and then for $18 \mathrm{~h}$ at $4^{\circ} \mathrm{C}$. After this and every subsequent step, the membranes were rinsed 3 times with TBS-T, pH 7.4. Subsequently, the membranes were incubated with the primary antibodies for $2.5 \mathrm{~h}$ at $37^{\circ} \mathrm{C}$, using the following dilutions: 1:2,000, 1:1,000, 1:500, and 1:250 in TBS. After washing, the membranes were incubated for $1.5 \mathrm{~h}$ at $37^{\circ} \mathrm{C}$ with the secondary antibodies conjugated with HRP, using the 1:2,000 dilution recommended by the manufacturer. 3-amino-9-ethylcarbazole (SigmaAldrich, St. Louis, USA) was used as a substrate for peroxidase. The density of the developed bands, proportional to AGE4 concentration, was determined using densitometry and was expressed in arbitrary units (AU). The readings were conducted using the Gel Doc $\mathrm{XR}^{+}$(Bio-Rad Laboratories), and the band density was analyzed using Image Lab software (Bio-Rad Laboratories). All detected signals were normalized against the background density of the membrane.

Except for determination of the coefficients of variation (CV\%), all samples were run in duplicate. Parallel control assays were performed, in which the incubation step with the primary antibodies was omitted to control for the specificity of the secondary antibodies. Any signals observed in the control assays were subtracted from the corresponding samples in the test assay.

\section{Statistical analysis}

Prior to the analysis, the results of the technical replicates were averaged. Data distribution was tested using the Kolmogorov-Smirnov test. Data on AGE4 is presented as medians with 95\% confidence intervals (95\% CI) and was analyzed using the Mann-Whitney U test. Normally distributed data was analyzed using the t-test for independent samples with Welch's correction if appropriate. The frequency analysis was conducted using Fisher's exact test. Comparisons of 2 proportions were conducted using $X^{2}$ statistics. Correlations were analyzed using Spearman's rank correlation coefficient. Stepwise logistic regression with $\mathrm{p}<0.05$ as inclusion and $\mathrm{p}>0.1$ as exclusion criteria was applied to identify independent predictors of AGE4 positivity or polyneuropathy. Odds ratios (ORs) with 95\% CI were calculated for significant variables. The accuracy of the model based on explanatory variables was determined using receiver operating characteristics (ROC) curve analysis and expressed as the area under the ROC curve (AUC). Additionally, the sensitivity and specificity of the model corresponding with the optimal cut-off were calculated.

All calculated $\mathrm{p}$-values were two-sided and $\mathrm{p} \leq 0.05$ was considered statistically significant. The statistical analysis was conducted using MedCalc Statistical Software v. 17.9.6 (MedCalc Software bvba, Ostend, Belgium).

\section{Results}

\section{Assay development}

\section{Competitive ELISA}

Our first attempt to develop an immunoassay for the detection and quantification of AGE4 was a competitive enzyme-linked immunosorbent assay (ELISA). In order 


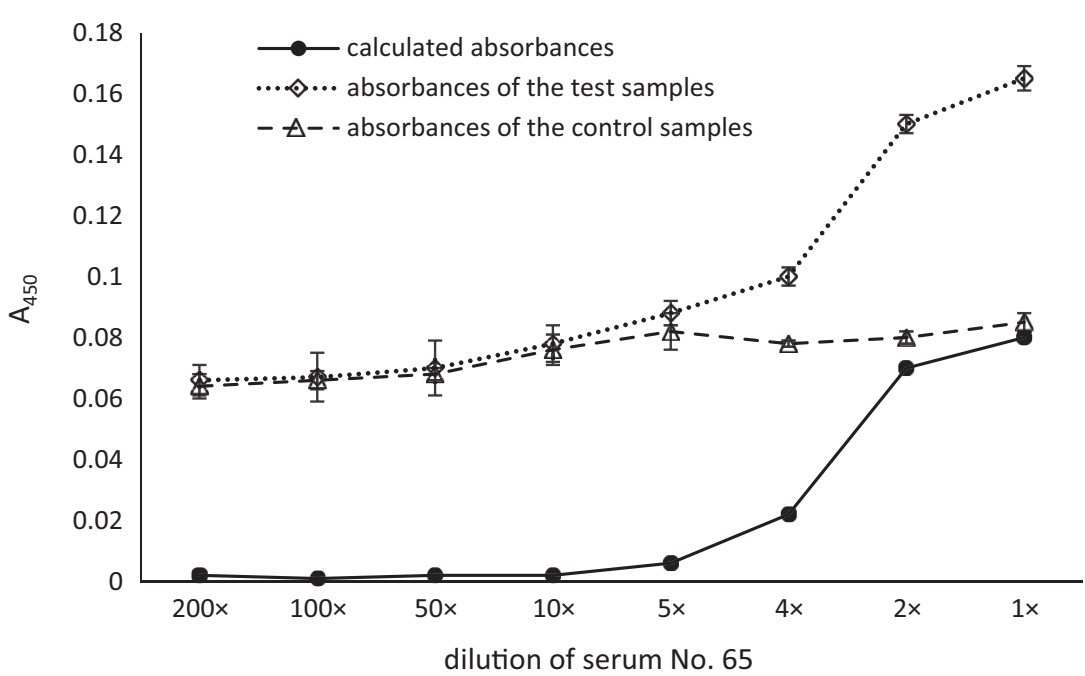

Fig. 1. The absorbance values of the test samples (serum No. 65), control samples and the calculated absorbance

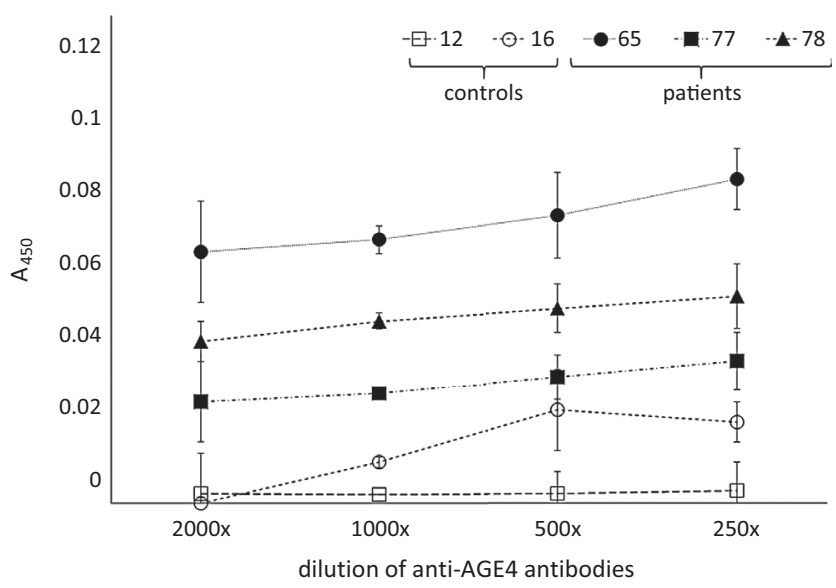

Fig. 2. Absorbance values (results after taking into account the absorbance values of the control samples, i.e., corresponding anti-AGE4 untreated sera) obtained using direct ELISA. Serum dilutions in carbonate buffer were $\times 1$. The anti-AGE4 dilution in TBS was $\times 250$

to establish the optimal assay conditions, microtiter plate wells were coated with different dilutions of serum samples, from undiluted sera up to 200-fold dilutions. Except for the least diluted sera, the readings recorded were almost identical with their respective controls (without anti-AGE4 antibodies). Therefore, when the control readings were subtracted from the samples, only the undiluted sera and 200-fold diluted sera had absorbance readings exceeding 0.05. An example of the effect of serum dilution on AGE4 detection is presented in Fig. 1.

The assay was further optimized by testing various dilutions of anti-AGE4 antibodies using plates coated with either undiluted or 200-fold diluted sera. Of the tested antibody dilutions, the highest sample-to-control absorbance ratios were obtained for the lowest dilution (1:250). There was no significant difference between assays conducted using undiluted or 200-fold diluted sera for the coating. The effect of antibody dilution factors on AGE4 detection is presented in Fig. 2 for 6 different sera (2 derived from healthy individuals and 4 from patients with diabetes).
Using the conditions yielding the highest sample-tocontrol readings, 23 randomly selected serum samples were used to detect and quantify AGE4. In 7 samples out of 23, reactions were clearly observed in anti-AGE4treated wells, yielding 30\% AGE4 positivity. Absorbance of the control wells (sera untreated by anti-AGE4) for these 7 samples was significantly lower. The median AGE4 absorbance was 0.118 (95\% CI $=0.13-0.09)$. The absorbance values for the samples were in the 0.06-0.17 range for $250 \times$ anti-AGE4 dilution and in the 0.06-0.09 range for corresponding control samples. Based on the results including the control test, it was found that trace amounts of AGE4 were detected in some sera, but the direct ELISA method was insufficiently sensitive.

\section{Slot dot-blot}

Since the control-adjusted readings in ELISA were generally low, the slot dot-blot method, which allows more antigens to be loaded on a membrane, was tried. The serum dilution was fixed at 1:200 and only the anti-AGE4 antibody step was adjusted. As in the ELISA test, the starting antibody dilution was 1:2,000. Since there was no signal on the membranes, lower dilutions were tested; a 1:250 dilution was found to yield signals in a number of sera (Fig. 3).

Using a 1:250 antibody dilution, 24 randomly selected sera were tested (22 from individuals with abnormalities in glucose metabolism and 2 from healthy blood donors). Simultaneously, a control assay, without anti-AGE4 antibodies, was run. A number of bands could be clearly observed in an anti-AGE4-treated blot: 17 samples out of 24, yielding $71 \%$ AGE4 positivity. However, there were only 2 responses in the control assay (Fig. 4, samples \#66 and \#68). The density of these control bands was relatively small, constituting $3.6 \%$ and $1.4 \%$ of the density recorded for corresponding samples treated with anti-AGE4. Nonspecific signals from the controls were subtracted from the test samples to calculate AGE4 levels. The median AGE4 level was 29,494 AU (95\% CI = 7,950-41,369). Data 

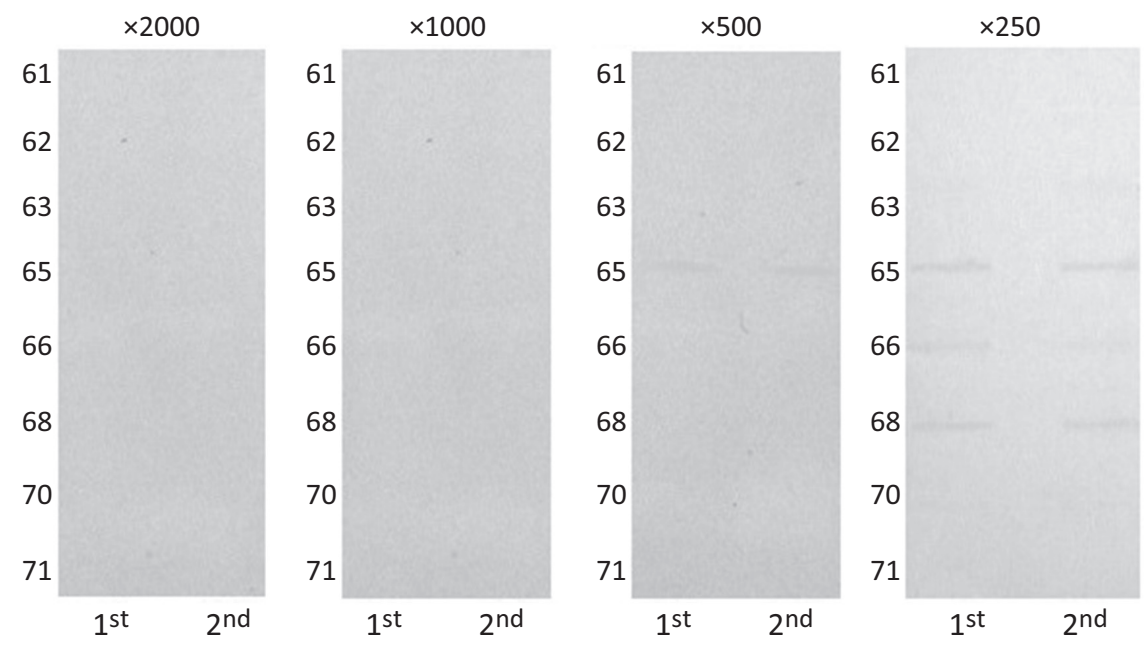

Fig. 3. Results of the slot dot-blot test carried out at the following anti-AGE4 dilutions: $\times 2,000, \times 1,000, \times 500$, and $\times 250$. The number of the sample tested is given on the left side of the blot and the number of the technical replicate is given at the bottom

blot with anti-AGE4 antibodies

control blot - without anti-AGE4

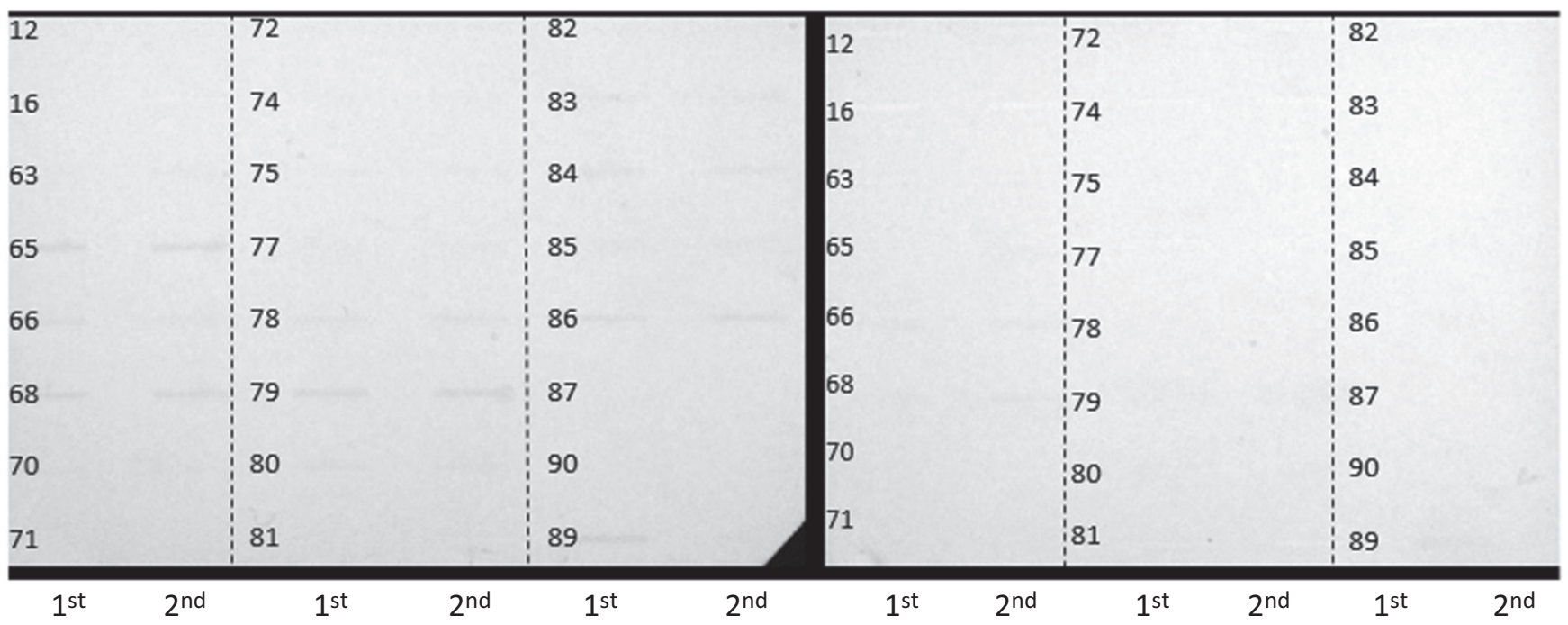

Fig. 4. Slot dot-blot results of diabetes sera treated with A) anti-AGE4 and secondary anti-mice lgG antibodies, and B) only secondary anti-mice IgG antibodies (control slot dot-blot). The number of sample tested is given on the left side of the blot and the number of the technical replicate is given at the bottom

on individual AGE4 levels for both assays (test and control) is presented in the Table 3. The mean CV\% calculated for technical replicates was $9.9 \%$.

In order to determine the intra- and inter-assay CV\%, 12 replicates of selected serum samples were run within the same assay on 9 separate runs. The calculated intraassay CV\% was $10.8 \%$ and the inter-assay CV\% was $10.6 \%$, both within the range acceptable for immunoassays.

The slot dot-blot method was used to quantify AGE4 levels in the healthy individuals and in the patients with diabetes with respect to disease complications, other metabolic abnormalities and treatment.

\section{AGE4 and diabetes}

As shown in Table 1, the healthy controls had lower AGE4 and AGE4 positivity than the patients. When analyzed in terms of the relationship with diabetes, the non-diabetic study participants had lower median AGE4 than the diabetics: 0 AU (0-0) vs 11,501 AU (3,061$26,905)(\mathrm{p}<0.0001)$ as well as lower AGE4 positivity $(19.4 \%$ vs $64.7 \%, \mathrm{p}<0.0001)$.

There was no significant difference between the diabetics and the controls in terms of median AGE4 ( $p=0.485)$ or AGE4 positivity ( $\mathrm{p}=0.992)$. However, the patients with uncontrolled disease had significantly lower median AGE4 than the patients with controlled disease (3,906 AU $(0-21,426)$ vs $26,979$ AU (6,605-45,322), $\mathrm{p}=0.033)$ and tended to have lower AGE4 positivity (58.2\% vs $76.6 \%, \mathrm{p}=0.102)$. There was also a negative correlation between AGE4 and $\mathrm{HbA}_{1} \mathrm{C}: \rho=-0.29$, $\mathrm{p}=0.008$, particularly in males: $\rho=-0.42, \mathrm{p}=0.018$.

\section{AGE4 and other metabolic abnormalities}

To evaluate correlations between AGE4 and various metabolic abnormalities, we compared the median AGE4 
Table 3. Band density of studied samples and controls. Errors of measurement

\begin{tabular}{|c|c|c|c|c|}
\hline $\begin{array}{l}\text { Serum } \\
\text { sample\# }\end{array}$ & $\begin{array}{c}\text { Band density } \\
\text { in test assay } \\
\text { (with } \\
\text { anti-AGE-4 } \\
\text { antibodies) } \\
\text { Mean [AU] }\end{array}$ & $\begin{array}{l}\mathrm{CV} \\
{[\%]}\end{array}$ & $\begin{array}{c}\text { Band density in } \\
\text { control assay } \\
\text { (without } \\
\text { anti-AGE-4 } \\
\text { antibodies) } \\
\text { Mean [AU] }\end{array}$ & $\begin{array}{l}\text { Calculated } \\
\text { AGE-4 } \\
\text { (test assay } \\
\text { - control } \\
\text { assay) } \\
\text { Mean [AU] }\end{array}$ \\
\hline 12 & 0 & - & 0 & 0 \\
\hline 16 & 0 & - & 0 & 0 \\
\hline 63 & 33,573 & 1.8 & 0 & 33,573 \\
\hline 65 & 82,128 & 3.6 & 0 & 82,128 \\
\hline 66 & 55,975 & 13.7 & 2,000 & 55,975 \\
\hline 68 & 81,450 & 1.4 & 1,100 & 81,450 \\
\hline 70 & 24,570 & 7.5 & 0 & 24,570 \\
\hline 71 & 53,975 & - & 0 & 53,975 \\
\hline 72 & 80,340 & - & 0 & 80,340 \\
\hline 74 & 26,650 & 16.4 & 0 & 26,650 \\
\hline 75 & 20,215 & 8.2 & 0 & 20,215 \\
\hline 77 & 34,060 & 13.8 & 0 & 34,060 \\
\hline 78 & 49,693 & 7.3 & 0 & 49,693 \\
\hline 79 & 84,858 & 7.8 & 0 & 84,858 \\
\hline 80 & 10,646 & 16.8 & 0 & 10,646 \\
\hline 81 & 0 & - & 0 & 0 \\
\hline 82 & 0 & - & 0 & 0 \\
\hline 83 & 38,545 & 11.7 & 0 & 38,545 \\
\hline 84 & 52,358 & 13.9 & 0 & 52,358 \\
\hline 85 & 38,220 & 10.8 & 0 & 38,220 \\
\hline 86 & 66,593 & 6.0 & 0 & 66,593 \\
\hline 87 & 16,315 & 17.3 & 0 & 16,315 \\
\hline 89 & 32,338 & - & 0 & 32,338 \\
\hline 90 & 0 & - & 0 & 0 \\
\hline
\end{tabular}

Measurement error was calculated as the ratio of absolute difference between 2 replicates to higher measurement and expressed as percent.

and the distribution of AGE4-positive samples among patients with and without obesity, hypertension, hyperlipidemia, hyperuricemia, fatty liver disease, and chronic kidney disease. Of these, obese patients had a significantly higher median AGE4 than non-obese ones: 33,573 AU $(15,786-46,033)$ vs $3,242 \mathrm{AU}(0-11,391)(\mathrm{p}=0.004)$ and a higher percentage of AGE4 positivity: $75.7 \%$ vs $55.6 \%$ $(\mathrm{p}=0.051)$. Moreover, there was a positive correlation between AGE4 and BMI: $\rho=0.27, p=0.018$.

\section{AGE4 and diabetic complications}

We also compared the median AGE4 and the distribution of AGE4-positive samples between patients with and without diabetic complications: macroangiopathy in general and as ischemic heart disease, acute coronary syndromes, ischemic stroke, arteriosclerosis obliterans, and carotid artery plaques; as well as microangiopathy in general and as retinopathy, nephropathy and neuropathy. AGE4 positivity tended to be less frequent in patients with microangiopathy in general as compared to patients without microangiopathy ( $54.3 \%$ vs $73.3 \%$; $\mathrm{p}=0.061)$. Detailed analysis showed a significant correlation between AGE4 positivity and polyneuropathy: Patients with this complication had significantly lower AGE4 positivity than those without it ( $45.7 \%$ vs $75 \%, \mathrm{p}=0.005)$.

\section{AGE4 and treatment}

We compared the median AGE4 and the distribution of AGE4-positive samples between patients treated and untreated with insulin, sulfonylurea, metformin, gliptins, acarbose, antihypertensive drugs, dyslipidemia medications, anticoagulants, aspirin, and clopidogrel. The median AGE4 was higher in metformin-treated than in non-treated patients: 20,215 AU $(6,533-35,430)$ vs 384 AU $(0-11,318)$ $(\mathrm{p}=0.011$ ). AGE4 positivity tended to be higher in metformin-treated patients as well $(69.2 \%$ vs $50 \%$ for non-treated; $\mathrm{p}=0.086$ ) but was significantly lower in gliptin-treated than non-treated patients $(33.3 \%$ vs $68.4 \%$; $=0.019)$.

\section{Independent predictors of AGE4 positivity: multivariate analysis}

To identify independent factors associated with AGE4 positivity, stepwise logistic regression was employed. Obesity, $\mathrm{HbA}_{1} \mathrm{C}$, polyneuropathy, and treatment with gliptins were entered as explanatory variables. Polyneuropathy (coefficient 1.55, $\mathrm{p}=0.003$ ) and obesity (coefficient 1.1, $\mathrm{p}=0.039$ ) were found independently associated with AGE4 positivity with the following odds ratios (ORs): 0.21 (95\% $\mathrm{CI}=0.08-0.58)$ for polyneuropathy and 3.02 (1.1-8.6) for obesity. The model built on both variables correctly classified $71.1 \%$ of the cases and its overall accuracy was $73 \%$.

When treatment with metformin was entered as an additional explanatory variable, it replaced obesity in the model. Polyneuropathy had a coefficient of $-1.67(p=0.002)$, while the coefficient for metformin was 1.33 ( $\mathrm{p}=0.027$ ). The ORs for polyneuropathy and metformin treatment were $0.19(0.07-0.53)$ and $3.76(1.16-12.18)$, respectively. The model correctly classified $70 \%$ of the cases and its accuracy was $72 \%$.

\section{AGE4 positivity as an independent predictor of polyneuropathy}

To test the strength of the association of AGE4 positivity with polyneuropathy, stepwise logistic regression was employed. To select variables associated with polyneuropathy in a univariate analysis, categorical data was tested using Fisher's exact test, while continuous data was submitted to a t-test for independent samples. Fasting glucose, $\mathrm{HbA}_{1} \mathrm{C}$, and triglycerides were significantly increased in patients with polyneuropathy. They were therefore entered into 
the model of logistic regression as explanatory variables in addition to AGE4 positivity. Of these, AGE4 positivity (coefficient $-1.42, \mathrm{p}=0.015$ ) and $\mathrm{HbA}_{1} \mathrm{C}$ (coefficient $0.37, \mathrm{p}=0.010$ ) were found to be independent predictors of polyneuropathy with ORs of 0.24 (95\% CI = 0.08-0.75) for AGE4 positivity and $1.6(1.1-2.3)$ for $\mathrm{HbA}_{1} \mathrm{C}$. The model built on both variables correctly classified $70.4 \%$ of the cases and its overall accuracy was $76 \%$. The combined markers were characterized by perfect specificity but low sensitivity in detecting polyneuropathy (Fig. 5).

\section{Discussion}

There is growing interest in detecting and quantifying individual AGE epitopes, as various AGEs may evoke different, even contradictory, responses in the body. ${ }^{23} \mathrm{How}-$ ever, the available tools remain limited. Of these, immunoassays are likely to have a potential for a widespread application due to their relative simplicity and low cost, but the available ELISAs for AGEs are usually universal assays not dedicated to quantifying individual AGEs epitopes. ${ }^{22,24}$

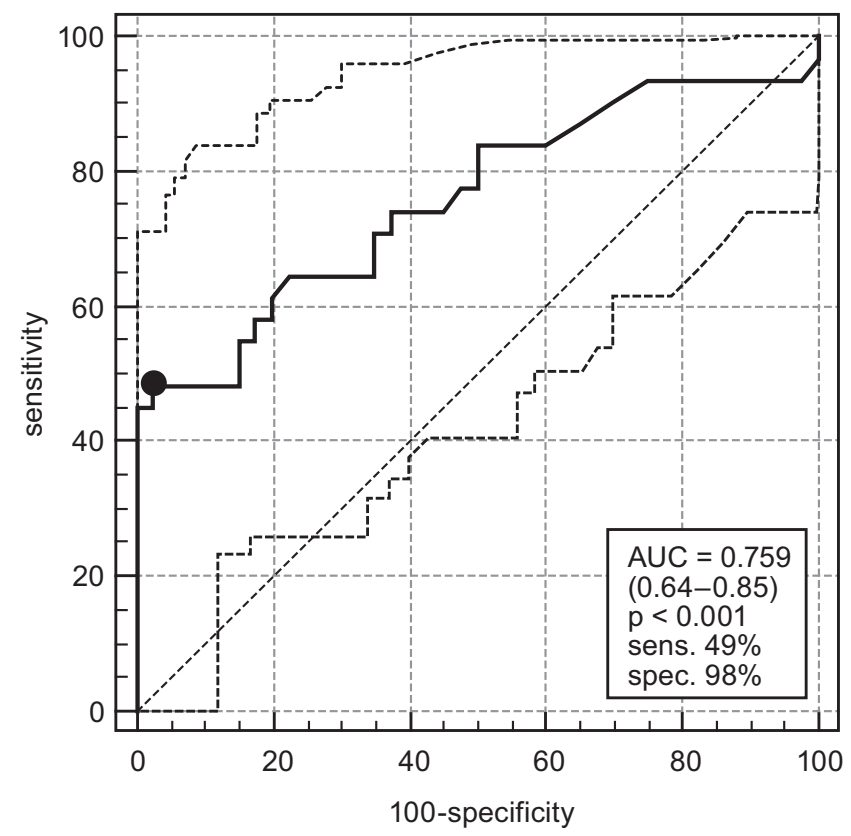

Fig. 5. Receiver operating characteristic (ROC) curve for combined AGE4 positivity and $\mathrm{HbA1C}$ as markers of polyneuropathy. Data is presented as area under ROC curve (AUC) with $95 \% \mathrm{Cl}$ and sensitivity (sens.) and specificity (spec.) corresponding with the optimal cut-off (represented as solid circle on the graph)

In this paper, we present a slot dot-blot method for the semi-quantitative determination of non-standard epitopes of AGE4 present in trace amounts in sera. In a classic ELISA using a microtiter plate format, the sensitivity of the assay is limited by the volume of the serum sample loaded into the well. The amount of antigen successfully bound to the microtiter plate well is directly correlated with its concentration in a medium. Therefore, the lower the concentration of an antigen of interest in the medium, the lower the probability of its being bound and not removed in subsequent washing steps. Moreover, the more complex the medium, the greater the presence of competing antigens, and the binding of a particular antigen of interest is even less probable. Although the problem can be solved by prior extraction of the antigen of interest, allowing for its relative concentration, such an approach is expensive and time- and labor-consuming. It may also require sophisticated procedures and equipment, thus hindering the widespread application of such a method in clinical and diagnostic laboratories. In this respect, the slot dot-blot method is superior, as it allows for more of the antigen of interest to be loaded on a carrier membrane. There is no volume limit of the serum sample to be loaded, and the use of a vacuum ensures that all the antigens are successfully bound and retained on the membrane. Therefore, as our study demonstrated, the slot dot-blot method allowed us, with satisfying reproducibility, to detect and semi-quantify AGE4 antigens undetectable with a competitive ELISA. The superior sensitivity of the slot dotblot method as compared to a classic ELISA has also been demonstrated by others, as it was previously used to detect the presence of malaria-causing Plasmodium falciparum in mosquitos. ${ }^{25,26}$

Using monoclonal antibodies to target non-standard epitopes of AGE4 (MGO-modified albumin) that do not cross-react with other AGEs or albumin modified by standard AGE epitopes such as CML and CEL, we showed that their increased serum levels correlate with diabetes and obesity. This observation is in line with AGE formation being accelerated not only by hyperglycemia but by hyperlipidemia, as well as by the oxidative stress and low-grade inflammation accompanying these conditions. ${ }^{27}$ Methylglyoxal represents low-molecular aldehydes derived from normal metabolism as well as pathological glycation and lipoxidation. The reactivity of MGO is about 20,000 times higher than the reactivity of glucose. As such, MGO has been called (by van der Heijden et al.) "a key compound involved in the very fast generation of glycation adducts", also produced in reactions with short-lived proteins. ${ }^{28} \mathrm{El}-$ evated concentrations of MGO in serum have previously been shown in diabetes ${ }^{20}$ and held responsible for inducing maladaptive responses in the vasculature. ${ }^{29}$ Similarly, MGO has been linked with diabetic complications, increasing risk of cardiovascular events and mortality, ${ }^{31}$ as well as impairing bone regeneration in patients with diabetes. ${ }^{32}$ Mechanistically, MGO has been shown to interfere with the platelet-derived growth factor B/platelet-derived growth factor receptor $\beta$ (PDGFB/PDGFR $\beta$ ) axis in smooth muscle cells and fibroblasts through formation of CML and CEL adducts on the PDGFB receptor. PDGFR $\beta$ inhibition, in turn, destabilizes the fibrous cap on atherosclerotic plaque, contributing to its rapture by inducing growth arrest and apoptosis of smooth muscle cells and fibroblasts. ${ }^{33}$ However, animal studies have shown that MGO-derived 
AGEs also accumulate in the pancreatic $\beta$ cells of healthy rats, and the levels of these AGEs were not affected by streptozotocin-induced diabetes. Therefore, it has been suggested that MGO-derived AGEs may play a positive role in the post-translational maturation of insulin. ${ }^{34}$

To our knowledge, there are no studies in the literature that analyze the amount of a specific epitope of an MGOmodified protein in the context of metformin; only the influence of metformin on MGO content in human serum has been checked. ${ }^{35}$ Beisswenger et al. used high-performance liquid chromatography to examine MGO in plasma from 57 subjects with T2DM, half of whom were being treated with metformin. Those authors found that metformin reduces $\mathrm{MGO}$ in a dose-dependent fashion and minimizes the effect of worsening glycemic control on MGO levels. ${ }^{36}$ A similar conclusion was reached by Kiho et al., who incubated BSA or RNase with MGO, and metformin turned out to be an inhibitor of AGE formation. ${ }^{37}$ In our study we found that the level of AGE4 is higher in patients treated with metformin. This is somewhat contradictory to the finding that the drug reduces systemic MGO levels in T2DM, because it is logical that the more MGO is present, the higher AGE4 levels are expected. Additional studies of the potential effects of metformin on MGO production and modifying proteins by MGO are required to further elucidate the role of metformin in diabetic complications.

The effect of gliptins (dipeptidyl peptidase-4 inhibitors, an established anti-inflammatory drug) on MGO-induced glycation has not been thoroughly researched. It has been reported that linagliptin improves MGO-induced peritoneal fibrosis. In a study carried out on mice, it was found that linagliptin suppressed the expression of fibrotic markers, such as alpha-smooth muscle actin ( $\alpha$-SMA), fibroblastspecific protein-1 (FSP-1) and type I and III collagen, and inhibited macrophage infiltration in MGO-induced peritoneal fibrosis. ${ }^{38}$ It has also been shown that gemigliptin inhibits MGO-modified AGE-BSA formation and traps MGO in a concentration-dependent manner. ${ }^{39}$ Regarding drugs of this group, our study found the expected results: AGE4 positivity was significantly lower in gliptin-treated patients than in non-treated patients, which confirmed its anti-glycation properties.

\section{Conclusions}

The extent to which high glucose levels in diabetic patients, as estimated by measuring $\mathrm{HbA}_{1} \mathrm{c}$ levels, contributes directly to vascular disease remains controversial. Recent studies have suggested that postprandial spikes of high glucose may be a more robust determinant of vascular risk than average glucose levels as determined by $\mathrm{HbA}_{1} \mathrm{c}$. However, we should recognize that $\mathrm{HbA}_{1} \mathrm{c}$ levels are unlikely to provide a fully accurate picture of temporal glycemic fluctuations, and thus there is a difference between diabetic individuals with and without regular occurrences of hyperglycemic spikes. This may be one of the underlying reasons why glycemic control, as measured by decreasing $\mathrm{HbA}_{1} \mathrm{c}$ levels, has not been consistent in successfully reducing vascular disease. ${ }^{40}$ The formation of MGO might be a mechanism that causes repeated glucose spikes to have a more damaging effect than high fasting or mean glucose levels on endothelial cells and the development of vascular complications. Methylglyoxal is a strong candidate for use as a more robust clinical marker of glycemic fluctuation than $\mathrm{HbA}_{1} \mathrm{c}$. A major challenge for the future would be to systematically analyze MGO-modified proteins in order to identify a better risk predictor than $\mathrm{HbA}_{1} \mathrm{c}$. Methylglyoxal triggers maladaptive responses in vascular tissue. ${ }^{41}$ AGE4 positivity and $\mathrm{HbA}_{1} \mathrm{c}$ were found to be independent predictors of polyneuropathy.

\section{ORCID iDs}

Agnieszka Bronowicka-Szydełko

(D) https://orcid.org/0000-0001-9967-036X

Małgorzata Krzystek-Korpacka (D) https://orcid.org/0000-0002-2753-8092 Aleksandra Kuzan (D) https://orcid.org/0000-0003-4264-8174 Kinga Gostomska-Pampuch (i) https://orcid.org/0000-0002-0771-3893 Małgorzata Gacka (D) https://orcid.org/0000-0001-5760-1534 Urszula Jakobsche-Policht (D) https://orcid.org/0000-0002-5510-2675 Rajmund Adamiec (D) https://orcid.org/0000-0002-5616-5088 Andrzej Gamian (D) https://orcid.org/0000-0002-2206-6591

\section{References}

1. Singh VP, Bali A, Singh N, Jaggi AS. Advanced glycation end products and diabetic complications. Korean J Physiol Pharmacol. 2014;18(1): 1-14.

2. Ott C, Jacobs K, Haucke E, Navarrete Santos A, Grune T, Simm A. Role of advanced glycation end products in cellular signaling. Redox Biol. 2014;2:411-429.

3. Singh R, Barden A, Mori T, Beilin L. Advanced glycation end-products. Diabetologia. 2001;44(2):129-146.

4. Goh SY, Cooper ME. Clinical review: The role of advanced glycation end products in progression and complications of diabetes. J Clin Endocrinol Metab. 2008;93(4):1143-1152.

5. Neelofar K, Arif Z, Ahmad J, Alam K. Non-enzymatic glucosylation induced neo-epitopes on human serum albumin: A concentration based study. PLoS One. 2017;13:2(2):e0172074.

6. Kaur N, Kishore L, Singh R. Dillenia indica L. attenuates diabetic nephropathy via inhibition of advanced glycation end products accumulation in STZ-nicotinamide induced diabetic rats. J Tradit Complement Med. 2017;8(1):226-238.

7. Bär KJ, Franke S, Wenda B, et al. Pentosidine and N- $\varepsilon$ carboxymethyl)lysine in Alzheimer's disease and vascular dementia. Neurobiol Aging. 2003;24(2):333-338.

8. de Vos LC, Lefrandt JD, Dullaart RP, Zeebregts CJ, Smit AJ. Advanced glycation end products: An emerging biomarker for adverse outcome in patients with peripheral artery disease. Atherosclerosis. 2016; 254:291-299.

9. Lopez-Clavijo AF, Duque-Daza CA, Soulby A, Canelon IR, Barrow M, O'Connor PB. Unexpected crosslinking and diglycation as advanced glycation end-products from glyoxal. J Am Soc Mass Spectrom. 2014; 25(12):2125-2133.

10. Haddad M, Knani I, Bouzidi H, Berriche O, Hammami M, Kerkeni M. Plasma levels of pentosidine, carboxymethyl-lysine, soluble receptor for advanced glycation end products, and metabolic syndrome: The metformin effect. Dis Markers. 2016;2016:6248264.

11. Hipkiss AR. On the relationship between energy metabolism, proteostasis, aging and Parkinson's disease: Possible causative role of methylglyoxal and alleviative potential of carnosine. Aging Dis. 2017;8(3): 334-345. 
12. Lin CC, Chan CM, Huang YP, Hsu SH, Huang CL, Tsai SJ. Methylglyoxal activates NF-KB nuclear translocation and induces COX-2 expression via a p38-dependent pathway in synovial cells. Life Sci. 2016;149: 25-33.

13. Jyot, Mir AR, Habib S, Siddiqui SS, Ali A, Moinuddin. Neo-epitopes on methylglyoxal modified human serum albumin lead to aggressive autoimmune response in diabetes. Int J Biol Macromol. 2016;86: 799-809

14. Mir AR, Moinuddin, Habib S, Khan F, Alam K, Ali A. Structural changes in histone $\mathrm{H} 2 \mathrm{~A}$ by methylglyoxal generate highly immunogenic amorphous aggregates with implications in auto-immune response in cancer. Glycobiology. 2016;26(2):129-141.

15. Wetzels S, Wouters K, Schalkwijk CG, Vanmierlo T, Hendriks J. Methylglyoxal-derived advanced glycation endproducts in multiple sclerosis. Int J Mol Sci. 2017;18(2). doi:10.3390/ijms18020421

16. Brunvand L, Heier M, Brunborg $C$, et al. Advanced glycation end products in children with type 1 diabetes and early reduced diastolic heart function. BMC Cardiovasc Disord. 2017;17(1):133.

17. WHO. Global report on diabetes. Geneva, Switzerland: World Health Organization; 2017.

18. WHO. Obesity: Preventing and managing the global epidemic. Report of a World Health Organization Consultation. WHO Technical Report Series 894. Geneva, Switzerland: World Health Organization; 2000.

19. Peralta C, Hamid P, Batool H, Al Achkar Z, Maximus P. Psoriasis and Metabolic Syndrome: Comorbidities and Environmental and Therapeutic Implications. Cureus. 2019;11(12):e6369. doi: 10.7759/cureus. 6369

20. Catapano AL, Graham I, De Backer G, et al. 2016 ESC/EAS Guidelines for the Management of Dyslipidaemias. Eur Heart J. 2016;37(39): 2999-3058.

21. Centers for Disease Control and Prevention. The Third National Health and Nutrition Examination Survey (NHANES III 1988-94) reference manuals and reports. Hyattsville, MD: National Center for Health Statistics; 1996.

22. Takeuchi M, Yanase $\mathrm{Y}$, Matsuura N, et al. Immunological detection of a novel advanced glycation end-product. Mol Med. 2001;7(11): 783-791.

23. Heier M, Margeirsdottir HD, Torjesen PA, Seljeflot I, Stensaeth $\mathrm{KH}$ Gaarder M. The advanced glycation end product methylglyoxalderivedhydroimidazolone-1 and early signs of atherosclerosis in childhood diabetes. Diab Vasc Dis Res. 2015;12(2):139-145.

24. Adisakwattana S, Thilavech T, Chusak C. Mesona chinensis Benth extract prevents AGE formation and protein oxidation against fructose-induced protein glycation in vitro. BMC Complement Altern Med. 2014;14:130.

25. Stone W, Grabias B, Lanke K, et al. A comparison of Plasmodium falciparum circumsporozoite protein-based slot blot and ELISA immuno-assays for oocyst detection in mosquito homogenates. Malar J. 2015;14(1):451.

26. Kumar S, Zheng H, Sangweme DT, et al. A chemiluminescent-western blot assay for quantitative detection of Plasmodium falciparum circumsporozoite protein. JImmunol Methods. 2013;390(1-2):99-105.
27. Khuhawar MY, Zardari LA, Laghari AJ. Capillary gas chromatographic determination of methylglyoxal from serum of diabetic patients by precolumn derivatization with 1,2-diamonopropane. J Chromatogr B Analyt Technol Biomed Life Sci. 2008;873(1):15-19.

28. van der Heijden RA, Bijzet J, Meijers WC, et al. Obesity-induced chronic inflammation in high fat diet challenged C57BL/6J mice is associated with acceleration of age-dependent renal amyloidosis. Sci Rep. 2015; 5:16474. doi:10.1038/srep16474

29. Mirza MA, Kandhro AJ, Memon SQ, Khuhawar MY, Arain R. Determination of glyoxal and methylglyoxal in the serum of diabetic patients by MEKC using stilbenediamine as derivatizing reagent. Electrophoresis. 2007;28(21):3940-3947.

30. Kandhro AJ, Mirza MA, Khuhawar MY. Capillary gas chromatographic determination of methylglyoxal from serum of diabetic patients by precolumn derivatization using meso-stilbenediamine as derivatizing reagent. J Chromatogr Sci. 2008;46(6):539-543.

31. Hanssen NMJ, Scheijen JLJM, Jorsal A, et al. Higher plasma methylglyoxal levels are associated with incident cardiovascular disease in individuals with type 1 diabetes: A 12-year follow-up study. Diabetes. 2017;66(8):2278-2283.

32. Aikawa T, Matsubara H, Ugaji S, et al. Contribution of methylglyoxal to delayed healing of bone injury in diabetes. Mol Med Rep. 2017;16(1):403-409.

33. Cantero AV, Portero-Otín M, Ayala V, et al. Methylglyoxal induces advanced glycation end product (AGEs) formation and dysfunction of PDGF receptor-beta: Implications for diabetic atherosclerosis. FASEB J. 2007;21(12):3096-3106.

34. Morioka Y, Teshigawara K, Tomono Y, et al. The specific localization of advanced glycation end-products (AGEs) in rat pancreatic islets. J Pharmacol Sci. 2017;134(4):218-224.

35. Peters AS, Wortmann M, Fleming TH, et al. Effect of metformin treatment in patients with type 2 diabetes with respect to glyoxalase 1 activity in atherosclerotic lesions. Vasa. 2019;48(2):186-192.

36. Beisswenger PJ, Howell SK, Touchette AD, Lal S, Szwergold BS. Metformin reduces systemic methylglyoxal levels in type 2 diabetes. Diabetes. 1999;48(1):198-202.

37. Kiho T, Kato M, Usui S, Hirano K. Effect of buformin and metformin on formation of advanced glycation end products by methylglyoxal. Clin Chim Acta. 2005;358(1-2):139-145.

38. Nagai T,Doi S, Nakashima A, etal.Linagliptin ameliorates methylglyoxalinduced peritoneal fibrosis in mice. PLoS One. 2016;11(8):e0160993

39. Jung E, Kim J, Kim SH, Kim S, Cho MH. Gemigliptin, a novel dipeptidyl peptidase-4 inhibitor, exhibits potent anti-glycation properties in vitro and in vivo. Eur J Pharmacol. 2014;744:98-102.

40. Prázný M, Škrha J, Šoupal J, Škrha J Jr. Glycemic variability and microvascular complications of diabetes. Cas Lek Cesk. 2017;156(6):308-313.

41. Beisswenger PJ, Howell SK, Russell GB, Miller ME, Rich SS, Mauer M. Early progression of diabetic nephropathy correlates with methylglyoxal-derived advanced glycation end products. Diabetes Care. 2013;36(10):3234-3239. 